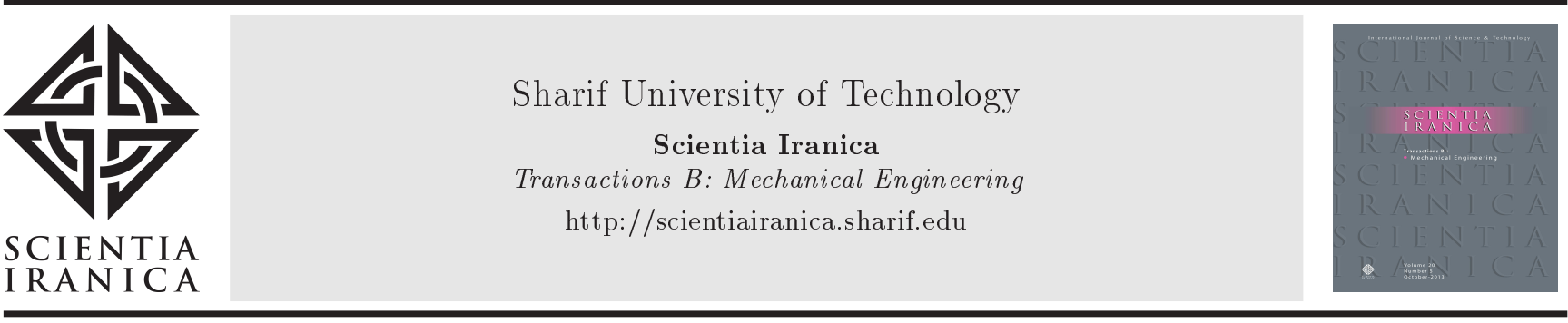

\title{
Multi-objective optimization of force convective heat transfer in a stack of horizontal channels using CFD and genetic algorithms: A comparison with asymptotic method results
}

\author{
H. Safikhani* and Z. Shirazi \\ Department of Mechanical Engineering, Faculty of Engineering, Arak University, Arak, P.O. Box 38156-88349, Iran.
}

Received 24 February 2016; received in revised form 3 September 2016; accepted 28 January 2017

\author{
KEYWORDS \\ Stack of horizontal \\ channels; \\ Force convection; \\ CFD; \\ Multi-objective \\ optimization; \\ Asymptotic method.
}

\begin{abstract}
In this paper, by combining the Computational Fluid Dynamics (CFD) and NSGA II algorithm, the forced convective heat transfer flow in a stack of horizontal parallel plates has been multi-objectively optimized. In the optimization process, the distance between the plates in the set of parallel channels has been changed so as to simultaneously optimize the amount of heat transfer between the plates and fluid and the pressure drop of the fluid (maximization of heat transfer and minimization of pressure drop). The Pareto front, which illustrates the changes of heat transfer from the plates and the pressure drop of fluid, simultaneously, has been presented in the results section. This result contains important information regarding the thermal designing of stack of channels subjected to forced convective heat transfer. The Pareto front has been obtained for four different fluids that have different Prandtl (Pr) numbers (mercury, air, water, and oil), and the results related to each fluid have been discussed. Finally, the multi-objective optimization results obtained in this paper have been compared with the results of the asymptotic analysis method (which is a single-objective method aimed at increasing the amount of heat transfer from plates) for internal fluid flows, and useful information has been obtained.
\end{abstract}

(C) 2018 Sharif University of Technology. All rights reserved.

\section{Introduction}

Heat exchangers are extensively used nowadays in various industries. One of the most frequently used types of heat exchangers is the plate heat exchanger. Today, with the diminishing of the energy resources, it is felt necessary to optimize these types of exchangers. Many investigations have been conducted in recent years on the numerical modeling, geometrical optimiza-

\footnotetext{
*. Corresponding author. Tel.: +98 8632625726 ;

Fax: +98 86 3262 5001

E-mail address: h-safikhani@araku.ac.ir (H. Safikhani)
}

tion, and the governing conditions of this type of heat exchanger [1-6]. Bejan and Sciubba [1] applied the asymptotic method to determine the optimum geometry of a stack of parallel plates cooled by forced fluid flow. They demonstrated that the thermal conditions of channel walls, with the temperature or flux being constant, did not led to much difference in the final results. They used a single-objective optimization, and their goal was to increase the amount of heat transfer in the stack of plates. By applying the genetic algorithms, Wei and Joshi [2] minimized the thermal resistance of a micro-channel heat sink. The design variables in their research consisted of some geometrical parameters including the blade thickness, ratio of channel width to blade thickness, etc. They 
performed their single-objective optimization process by considering two constraints: maximum permitted pressure loss and maximum permitted inlet flow rate. By combining numerical, analytical, and experimental studies and employing the asymptotic analysis method, Bejan et al. [3] optimized the natural convective flow around a stack of horizontal cylinders. They used a single-objective optimization approach; the design variable in their research was the distance between the cylinders and the constraint they had in mind was the constant volume of the region under investigation. Finally, they presented an equation for the optimal distance between the cylinders and the maximum degree of heat transfer, which was a function of geometrical variables and the non-dimensional Rayleigh number. Goshayeshi and Ampofo [4] numerically investigated the performance of heat sinks, placed horizontally and vertically and subjected to natural convective flow. They concluded that the vertical heat sinks had the best performance. Bar-Cohen and Rohsenow [5] used the analytical equations to calculate the optimum channel width for the maximum amount of heat transfer in a stack of vertical channels subjected to natural convection, for two boundary conditions of constant temperature and constant flux at channel walls. Using numerical modeling, Buonomo and Manca [6] explored the flow of air in vertical microchannels subjected to free convection. They investigated the slipping fluid flow, and the wall boundary condition was the constant flux at the walls. Some other researchers investigated optimization methods in heat transfer engineering applications [7-12].

In recent years, some researchers have used novel optimization methods in engineering applications [1315]. Hao et al. [13] investigated surrogate-based optimum design for stiffened shells with adaptive sampling. They investigated two illustrative examples to demonstrate the efficiency of the surrogate-based optimization method. One was a three-dimensional rigid-frame model, and the optimizations using the proposed method, direct surrogate-based optimization, and multi-island genetic algorithm were compared based on this model. The other was a typical fuel tank model configured for use in the booster of a launch vehicle. Three adaptive sampling strategies with different distributions of sampling points were compared with the direct surrogate-based optimization. Results indicated that the bi-step surrogatebased optimization with adaptive sampling provided an efficient and practical approach to find the optimum design for a structure with numerous variables and various types.

In the heat exchangers performed by force convection, it would be ideal to have a maximum heat transfer rate and minimum pressure drop. In fact, the heat transfer and pressure drop conflict with each other; thus, to achieve the optimal thermal behaviors in these channels, an MOO approach should be used to discover the best possible design points with appropriate heat transfer and pressure drop. NSGA II algorithm is one of the best and most complete multi-objective optimization algorithms, which is used in this paper as well. This algorithm was first proposed by Deb et al. [16], and it has been used in recent years in various engineering-related applications [17-19].

To the best of the authors' knowledge, a multiobjective optimization using the combination of Computational Fluid Dynamics (CFD) and genetic algorithm has not been utilized yet for the optimization of a stack of parallel plates with different fluids (different Prandtl numbers) passing through them. In this paper, by combining the CFD and NSGA II algorithm, the force convective heat transfer flow in a stack of horizontal parallel plates has been multi-objectively optimized. In the optimization process, the distance between the plates in the set of parallel channels has been changed so as to simultaneously optimize the amount of heat transfer between the plates and fluid and the pressure drop of the fluid (maximization of heat transfer and minimization of pressure drop).

\section{Mathematical modeling}

The data needed for performing the multi-objective optimization in this paper have been obtained by means of numerical modeling. The details of this procedure will be described in this section.

\subsection{Geometry}

The geometry investigated in this paper has been schematically illustrated in Figure 1. As shown in this figure, a number of parallel plates have been placed next to each other, which form a stack of parallel channels. Since, in this stack, the fluid flow field in each channel is the same, the governing equations have been numerically solved for one channel of width $D$ and length $L$; and, finally, the total heat transfer from the

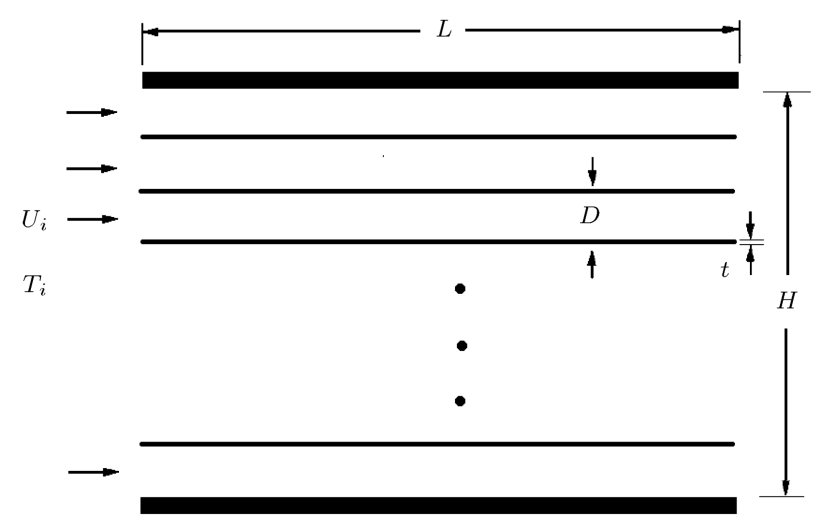

Figure 1. Schematic of a stack of horizontal channels cooled by force convection. 
Table 1. Some parameters and operating conditions used in the numerical simulations.

$$
\begin{aligned}
& 0.004 \leq D / L \leq 1 \\
& H / L=1 \\
& t \cong 0 \\
& \operatorname{Re}=1000 \\
& \operatorname{Pr}=0.01,0.72,7,13000 \\
& T_{i}=20^{\circ} \mathrm{C} \\
& T_{w}=75^{\circ} \mathrm{C}
\end{aligned}
$$

plates and pressure drop have been multiplied by the number of channels to get the total values. The total pressure drop and heat transfer for the set of channels are computed as follows:

$$
\begin{aligned}
& q_{\mathrm{tot}}=n q_{1}, \\
& \Delta P_{\mathrm{tot}}=\Delta P_{1}, \\
& n \approx \frac{L}{D} .
\end{aligned}
$$

The thickness of the plates has been ignored in the computations $(t \approx 0)$. In fact, the problem in this paper is defined as: "How many channels can be established on a plate of specific dimensions $(L \times H)$ so that the pressure drop of fluid and heat transfer from the stack of plates are simultaneously optimized?" Some of the constants and specifications used in this paper have been presented in Table 1.

\subsection{Governing equations}

In this paper, the governing equations constitute the continuity, Navier-Stokes, and energy equations in steady and laminar flow regimes $(\operatorname{Re}=1000<2300)$. These equations are expressed as:

- Continuity equation:

$$
\nabla \cdot(\rho \vec{V})=0 .
$$

- Momentum equation:

$$
\nabla \cdot(\rho \vec{V} \vec{V})=-\nabla P+\nabla \cdot(\mu \nabla \vec{V}) .
$$

- Energy equation:

$$
\nabla \cdot\left(\rho \vec{V} C_{P} T\right)=\nabla \cdot(k \nabla T) .
$$

\subsection{Boundary conditions}

For numerical simulation, the equations of the previous sections should be solved subject to the following boundary conditions:

- Channel inlet:

$$
\begin{aligned}
& V_{x}=V_{i}, \quad V_{y}=0, \\
& T=T_{i} .
\end{aligned}
$$

- Fluid-wall interface:

$$
\begin{aligned}
& V_{x}=V_{y}=0, \\
& T=T_{W} .
\end{aligned}
$$

- Channel outlet:

$$
P=P_{\text {atm }} \text {. }
$$

The values of all the variables have been adjusted so that for fluids with different Pr numbers, all the nondimensional parameters (e.g., Reynolds number) are equal to each other.

\subsection{Numerical methods}

The numerical simulation is performed using the finite volume method. A second order upwind method is used for the convective and diffusive terms and the SIMPLE algorithm is employed to solve the coupling between the velocity and pressure fields. The computations are continued until the solution converges with residuals of less than $10^{-8}$.

The mesh generation algorithm has been designed so that for every channel an appropriate non-uniform structured mesh is automatically generated. Due to the high gradient of the parameters, the generated elements at channel inlet and near the walls have finer sizes. The examined channels contain between 100 to 150 thousand structured elements. Figure 2 shows a sample of grid generation for computational domain in the present study. To make sure that the obtained results are independent of the size and the number of generated grids, several grids with different sizes along different directions have been tested for each structure; and it has been tried to consider the best grid, with the highest accuracy and the lowest computation cost, for each structure. Figure 3 shows the results of Grid Independency test (GI test) for 4 different generated grids. As shown in Figuer 3(a), all of the generated

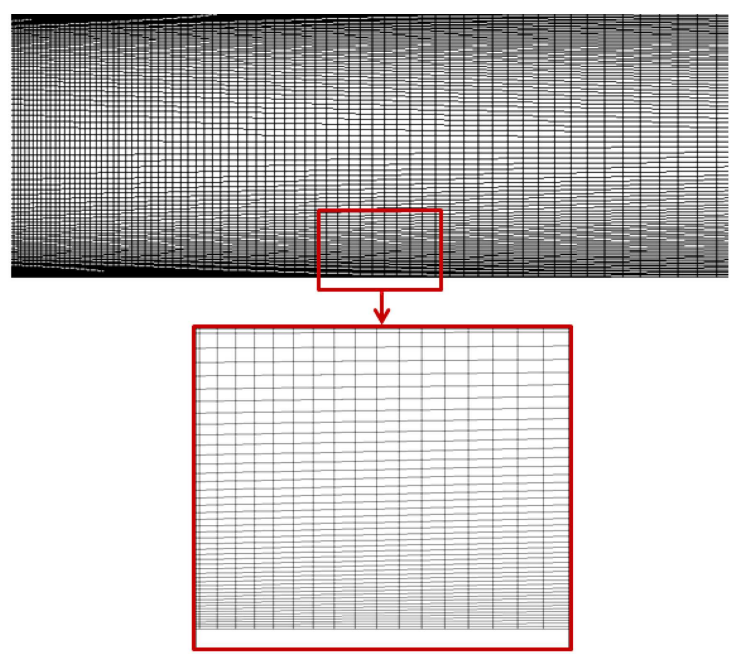

Figure 2. Structured non-uniform grid generations performed in the present paper. 


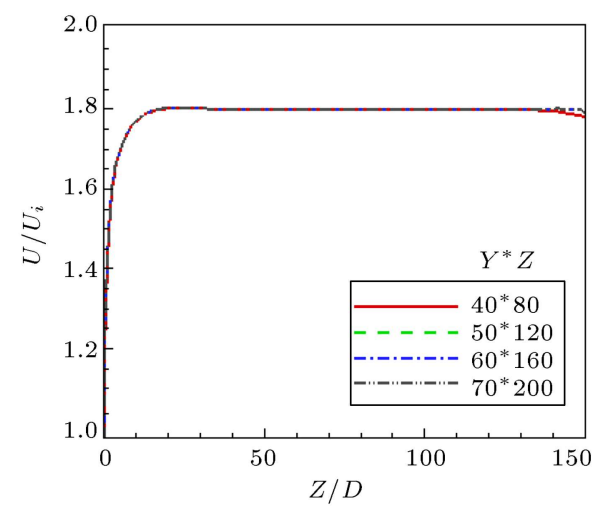

(a)

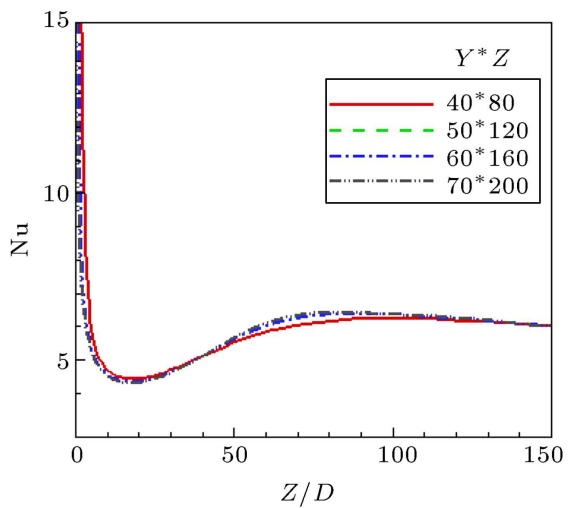

(b)

Figure 3. Grid independency test: (a) Axial velocity at center line, and (b) local Nu.

grids pass the GI test for dimensionless axial velocity $\left(U / U_{i}\right)$. The generated grid with $40 \times 80$ number of nodes in $y$ and $z$ directions, respectively, fails in GI test of $\mathrm{Nu}$ (Figure 3(b)), therefore the grid with $50 \times 120$ number of nodes in $y$ and $z$ directions, respectively, is used in the simulations. It should be noted that the generated meshes may be distorted during the optimization process due to the changes in the distance between plates.

\subsection{Validations}

To be sure of the validity of the numerical modeling used in the optimization process, the obtained numerical results should be compared with similar and available data. In Figure 4, the local $\mathrm{Nu}$ number computed in this paper is compared with similar values obtained by [20]. As observed in Figure 4, the Nu number computed in this paper accurately complies

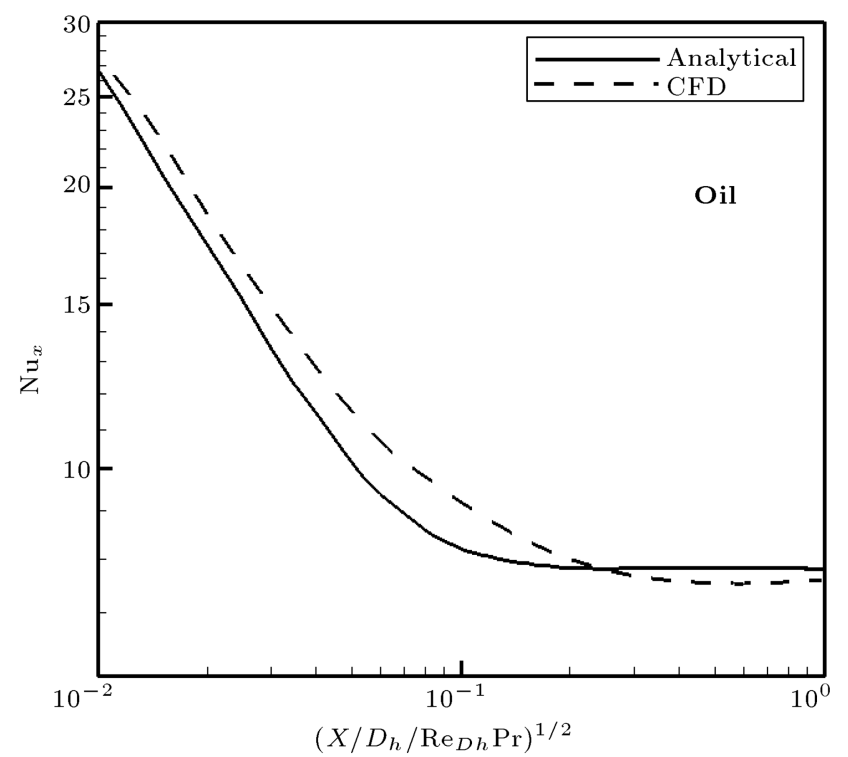

Figure 4. Comparison of local $\mathrm{Nu}$ of the present numerical calculation with the similar available data. with those obtained by other researchers; therefore, these data can be used in the optimization process.

\section{Results}

In order to investigate the optimal cooling performance of stack of horizontal channels, the data of the numerical simulations presented in Section 2 are now employed in a multi-objective optimization procedure using NSGA II algorithms [16]. In all runs, a population size of 60 has been chosen with crossover probability $\left(P_{c}\right)$ and mutation probability $\left(P_{m}\right)$ of 0.7 and 0.07 , respectively. The two conflicting objectives are $q_{\text {tot }}$ (heat transfer from stack of channels) and $\Delta P$ (pressure drop of fluid passing from stack) that should be optimized simultaneously with respect to the design variables $D$ (or number of channels). The multiobjective optimization problem can be formulated in the following form:

$$
\begin{cases}\text { Maximize } & q_{\mathrm{tot}}=f_{1}(D) \\ \text { Minimize } & \Delta P_{\mathrm{tot}}=f_{2}(D) \\ \text { Subject to } & 0.004 \leq D / L \leq 1\end{cases}
$$

Figure 5 shows the Pareto front for each of the four fluids with different $\operatorname{Pr}$ numbers obtained for the mentioned two objective functions. As this figure indicates, for each fluid, the points in the Pareto front have no dominancy over one another, i.e. no two points could be found with one having objective functions equal to each other and another having objective functions different from each other. In other words, if we move from one optimal point to another, one objective function will definitely improve and another objective function will certainly get worse. Although all the points in this Pareto front are optimal points, five points with special and unique characteristics are also observed, which have been designated as points $\mathrm{A}, \mathrm{B}, \mathrm{C}, \mathrm{D}$, and $\mathrm{E}$. The details of the design variables, 

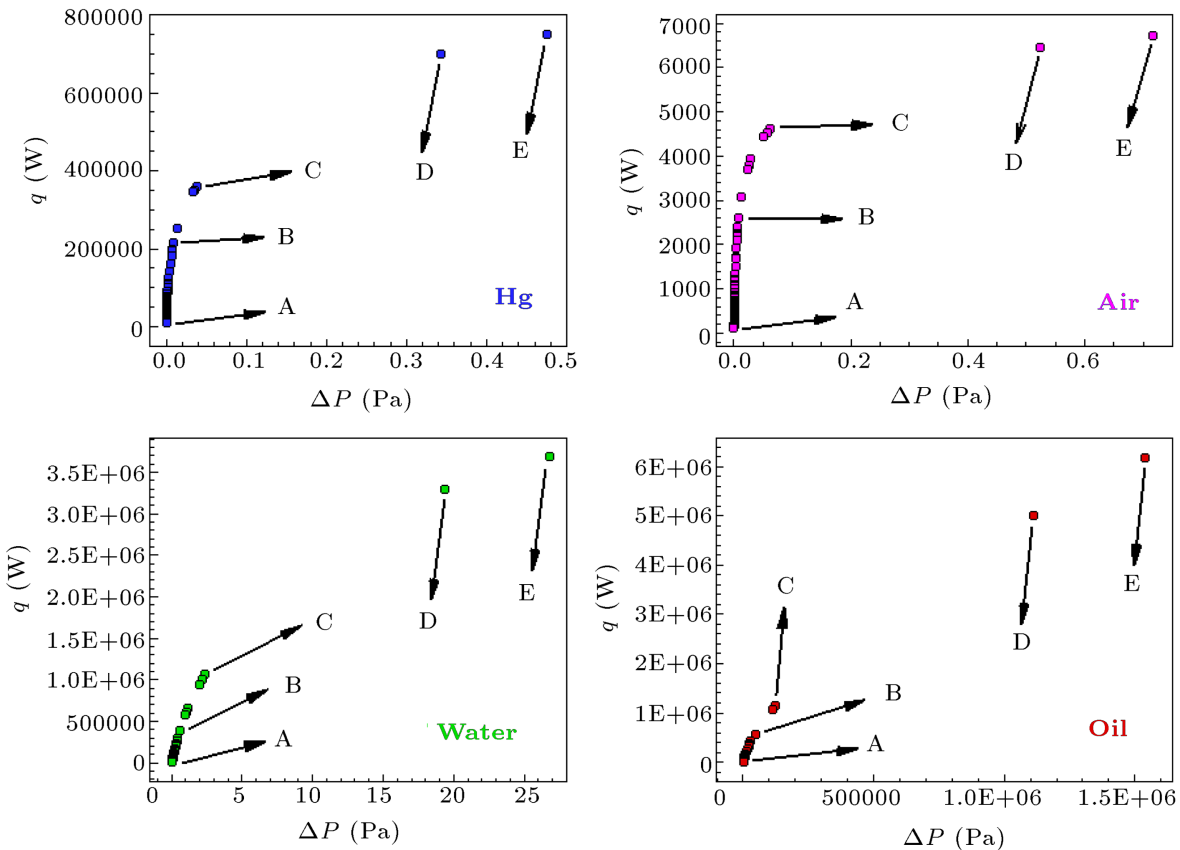

Figure 5. Pareto optimal points for $q_{\text {tot }}$ and $\Delta P$ of the four different fluids.

objective function values, and some of the other important parameters of these five optimal points have been shown in Table 2. Points $\mathrm{A}$ and $\mathrm{E}$ display the best $\Delta P$ and $q$, respectively.

Points B and D, known as the break points, are also interesting points in the design. In fact, as we go from point $\mathrm{A}$ to point $\mathrm{B}, \Delta P$ increases very slightly, while $q$ increases considerably. Similarly, as we go from point E towards point $\mathrm{D}, q$ increases slightly, while $\Delta P$ improves by a higher value.

In general, an optimal point in thermal design of stack of channels is a point where both of the objective functions are equally satisfied. In this paper, the mapping method [17] is used to compute and find such a point. In this method, both objective functions are mapped between 0 and 1 and, then, the norm of their sum is calculated. A point with the highest norm is the point at which both objective functions have been optimized to the same value. In this paper, point $\mathrm{C}$ has been determined by using the mapping methods, and both objective functions are equally satisfied at this point.

Figure 6 shows the overlay of Pareto fronts obtained for the four different fluids (mercury, air, water, and oil). An interesting point indicated by these diagrams is that the variation ranges of heat transfer and pressure drop values for oil are much greater than those for the other three fluids. This demonstrates that oil can be suitably used for the cooling of different industrial equipment, and that the high pressure loss of oil can be compensated by means of giant pumps.

The trend of changes of the objective functions with respect to the design variables in the Pareto

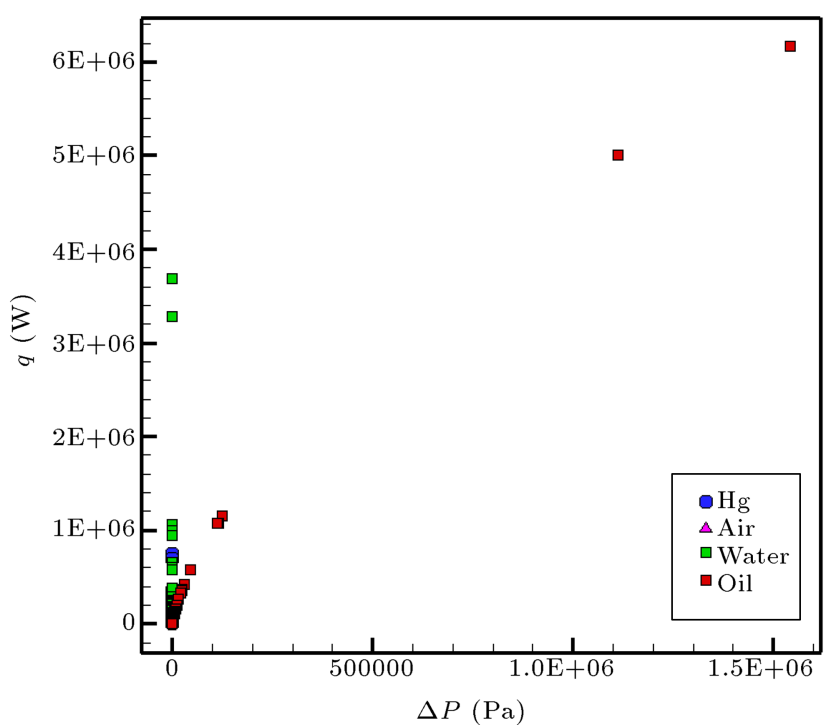

Figure 6. Overlay of Pareto optimal points of the four different fluids.

front conveys important and valuable information. The changes of pressure drop and heat transfer versus the changes of channel width have been illustrated in the Pareto diagrams in Figure 7 for the mentioned four fluids. The variations of heat transfer and pressure drop have been indicated by triangles and circles, respectively. As shown in this figure, for small values of $D / L$, the heat transfer and pressure drop changes are negligible; however, the values of heat transfer and pressure drop increase, and the degree of their increase depends on the value of the Pr number.

In addition to evaluating and comparing the 
Table 2. The values of objective functions and the associated design variables of the optimum points.

\begin{tabular}{|c|c|c|c|c|c|c|c|}
\hline \multirow{2}{*}{ Fluid } & \multirow{2}{*}{ Points } & \multirow{2}{*}{$\begin{array}{c}\text { Design variables } \\
D / L \\
\end{array}$} & \multicolumn{2}{|c|}{ Objective functions } & \multicolumn{3}{|c|}{ Other important variables } \\
\hline & & & $q_{\text {total }}(\mathbf{W})$ & $\Delta P(\mathrm{~Pa})$ & $\Delta T(\mathbf{K})$ & $n \cong \frac{H}{D}$ & $q_{1}(\mathrm{~W})$ \\
\hline \multirow{5}{*}{ Mercury } & A & 1.0000 & 10969 & 0.00014 & 39.85 & 1 & 10969 \\
\hline & $\mathrm{B}$ & 0.0310 & 214986 & 0.00958 & 75.00 & 32 & 6718.31 \\
\hline & $\mathrm{C}$ & 0.0150 & 361215 & 0.03891 & 75.00 & 66 & 5472.95 \\
\hline & $\mathrm{D}$ & 0.0050 & 698117 & 0.34278 & 75.00 & 199 & 3508.12 \\
\hline & $\mathrm{E}$ & 0.004 & 750555 & 0.47523 & 75.00 & 234 & 3207.50 \\
\hline \multirow{5}{*}{ Air } & $\mathrm{A}$ & 1.0000 & 113 & 0.00025 & 13.87 & 1 & 113.00 \\
\hline & $\mathrm{B}$ & 0.0451 & 2594 & 0.00798 & 75.00 & 22 & 117.90 \\
\hline & $\mathrm{C}$ & 0.0167 & 4453 & 0.05152 & 75.00 & 59 & 75.47 \\
\hline & $\mathrm{D}$ & 0.0051 & 6447 & 0.52370 & 75.00 & 192 & 33.57 \\
\hline & $\mathrm{E}$ & 0.004 & 6730 & 0.71717 & 75.00 & 234 & 29.91 \\
\hline \multirow{5}{*}{ Water } & A & 1.0000 & 10523 & 0.02199 & 5.09 & 1 & 10523.00 \\
\hline & B & 0.0350 & 386289 & 0.58930 & 24.17 & 28 & 13796.03 \\
\hline & C & 0.0150 & 1061020 & 2.35300 & 43.39 & 66 & 16076.06 \\
\hline & $\mathrm{D}$ & 0.0050 & 3287480 & 19.3515 & 75.00 & 199 & 16520.00 \\
\hline & $\mathrm{E}$ & 0.004 & 3686820 & 26.7564 & 75.00 & 234 & 15755.64 \\
\hline \multirow{5}{*}{ Oil } & A & 1.0000 & 5664 & 355.608 & 0.62 & 1 & 5664.00 \\
\hline & B & 0.0250 & 574158 & 46314.8 & 5.53 & 39 & 14722.00 \\
\hline & $\mathrm{C}$ & 0.0150 & 1152860 & 125626 & 8.01 & 66 & 17467.57 \\
\hline & $\mathrm{D}$ & 0.0050 & 4999250 & 1110870 & 17.24 & 199 & 25121.85 \\
\hline & $\mathrm{E}$ & 0.004 & 6166560 & 1540320 & 19.36 & 234 & 26352.82 \\
\hline
\end{tabular}
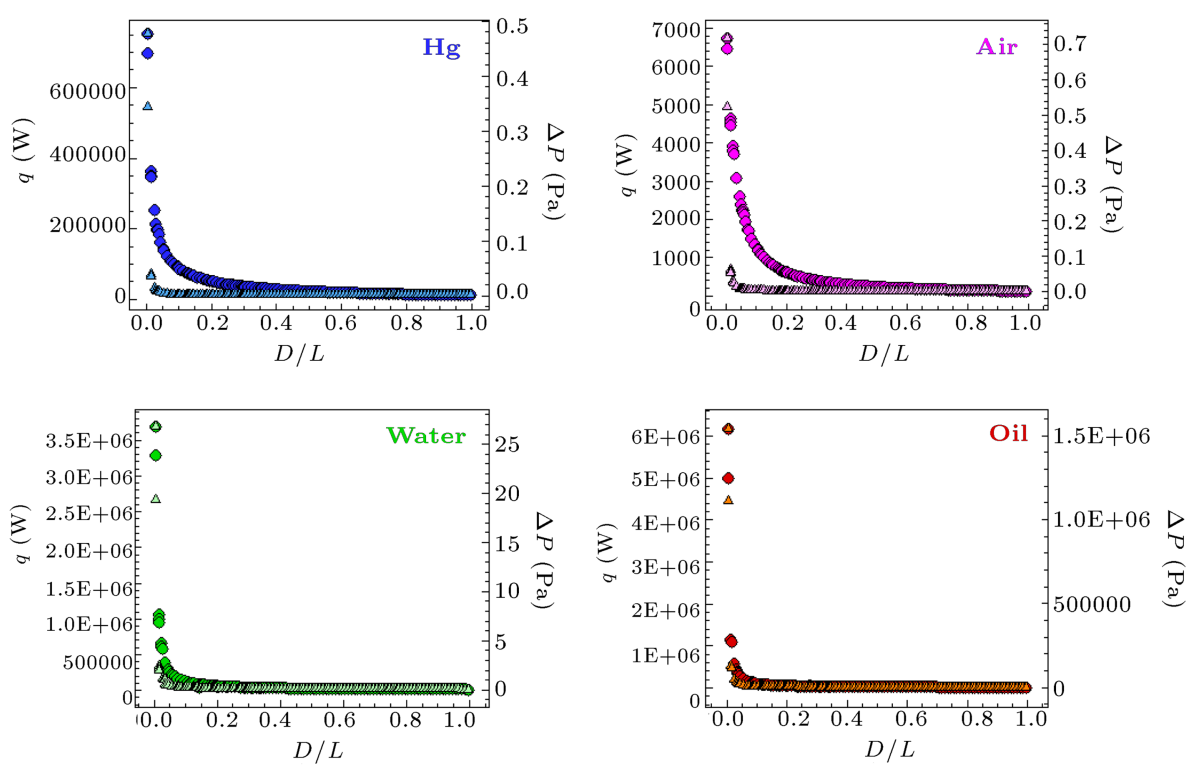

Figure 7. Optimal variations of $q_{\text {tot }}$ (triangle) and $\Delta P$ (circle) with respect to $D / L$ for four different fluids. 


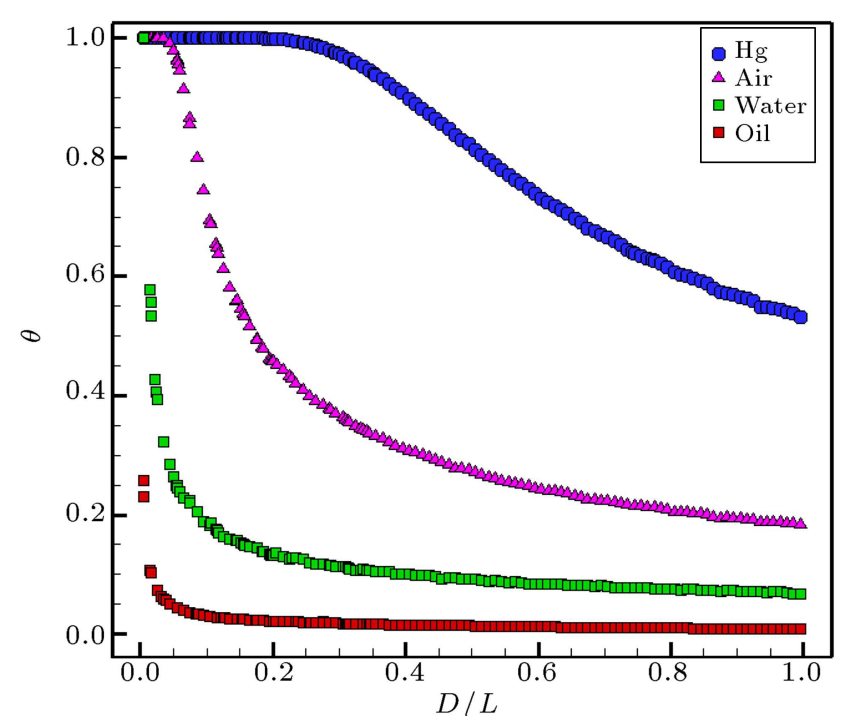

Figure 8. Optimal variations of averaged dimensionless temperature at the channel outlet with respect to $D / L$ for four different fluids.

variations of the heat transfer and pressure drop values for the four different fluids, it is important to examine the changes of the outlet fluid temperature. The four mentioned fluids, due to having different Pr numbers, exhibit different thermal behaviors. Figure 8 shows the averaged non-dimensional outlet temperatures of the four different fluids versus channel width in the Pareto front. As it is observed, the outlet temperature of mercury (with the least Pr number among the mentioned fluids), becomes equal to wall temperature at all the $D / L$ values less than 0.25 . Conversely, the outlet temperature of oil, which has a very high Pr number, does not become equal to wall temperature, even at the lowest value of $D / L$. This causes the physical properties of oil (which are temperature-dependent) not to change so easily. This characteristic of oil, i.e. change in its temperature being more difficult than that in other fluids, along with its previously mentioned property, i.e. high heat transfer capacity, has led to the extensive use of oil in different industries.

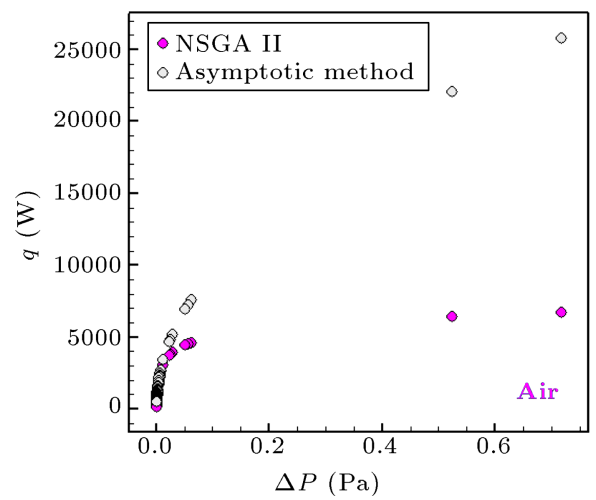

Using the asymptotic optimization method, Bejan $[1,20]$ obtained and reported the optimum channel width and heat transfer values in the forced convective flow process as:

$$
\begin{aligned}
& D_{\text {opt }} / L \cong 2.73 \mathrm{Be}_{L}^{\frac{-1}{4}}, \\
& q_{\max }=0.62\left(\frac{\rho \Delta P}{\operatorname{Pr}}\right)^{\frac{1}{2}} H C_{P}\left(T_{W}-T_{\infty}\right),
\end{aligned}
$$

where $\mathrm{Be}_{L}$ is Bejan number and is defined as $\mathrm{Be}_{L}=$ $\frac{\Delta P L^{2}}{\mu \alpha}$. The results obtained by Bejan et al. $[1,20]$ using the asymptotic approach have been compared in Figure 9 with the Pareto fronts obtained in this paper for air and water fluids. As it is observed, there is a relatively large difference between the results of the asymptotic method and the method presented in this paper. The reason is that the asymptotic method is not actually a classical optimization approach. In fact, no optimization takes place in this method, and only the behaviors of the internal and external flows are equalized at this point. The MOO method presented in this paper has a lot of advantages over the asymptotic method; e.g., the MOO method in this paper is CFD based; thus, it has higher accuracy. Moreover, it is a classic optimization method unlike asymptotic method. The other advantage of the presented method is the ability to recognize the break points (points $B$ and D in this paper) in Pareto front, which is only possible in multi-objective optimization methods.

\section{Conclusions}

In this paper, by combining CFD and NSGA II algorithm, the forced convective heat transfer flow in a stack of horizontal parallel plates was multiobjectively optimized. In the optimization process, the distance between the plates in the set of parallel channels was changed so as to simultaneously optimize the amount of heat transfer between the plates and fluid and the pressure drop of the fluid (maximization

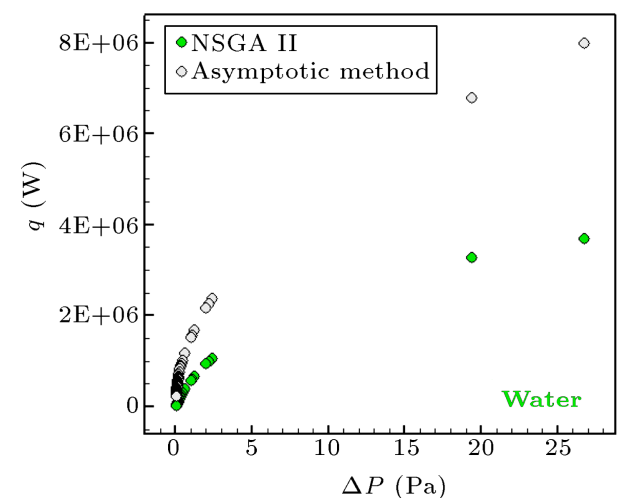

Figure 9. Overlay of Pareto optimal points calculated in present paper related to water and air and asymptotic method results. 
of heat transfer and minimization of pressure drop). The Pareto front, which illustrates the changes of the heat transfer from the plates and the pressure drop of fluid, simultaneously, was presented in the results section. This result contained very important information regarding the thermal designing of stack of channels subjected to forced convective heat transfer. The Pareto front was obtained for four different fluids that had different Prandtl (Pr) numbers (mercury, air, water, and oil), and the results related to each fluid were discussed. Finally, the multi-objective optimization results obtained in this paper were compared with the results of the asymptotic analysis method (which is a single-objective method aimed at increasing the amount of heat transfer from plates) for internal fluid flows; and very useful and valuable information was obtained.

\section{Nomenclature}

$C_{p} \quad$ Specific heat $\left(\mathrm{Jkg}^{-1} \mathrm{~K}^{-1}\right)$

$D \quad$ Width of one channel (m)

$H \quad$ Width of the main channel (m)

$h \quad$ Local heat transfer coefficient $\left(\mathrm{W} / \mathrm{m}^{2} \mathrm{~K}\right)$

$\bar{h} \quad$ Mean heat transfer coefficient $\left(\mathrm{W} / \mathrm{m}^{2} \mathrm{~K}\right)$

$k \quad$ Thermal conductivity $\left(\mathrm{Wm}^{-1} \mathrm{~K}^{-1}\right)$

$L \quad$ Length of channels (m)

$\mathrm{Nu} \quad$ Nusselt number $\left(=h D_{h} / k\right)$

$P \quad$ Pressure $(\mathrm{Pa})$

Pr Prandtl number $(=\alpha / \nu)$

$q \quad$ Heat transfer rate $(\mathrm{W})$

$q^{\prime \prime} \quad$ Heat flux $\left(\mathrm{W} / \mathrm{m}^{2}\right)$

Re Reynolds number $\left(=\rho V D_{h} / \mu\right)$

$T$ Temperature (K)

$V \quad$ Velocity $\left(\mathrm{ms}^{-1}\right)$

$x \quad$ Axial coordinate

\section{Greek symbol}

$\mu \quad$ Dynamic viscosity $\left(\mathrm{Nsm}^{-2}\right)$

$\nu \quad$ Kinematic viscosity $\left(\mathrm{m}^{2} \mathrm{~s}^{-1}\right)$

$\rho \quad$ Density $\left(\mathrm{kgm}^{-3}\right)$

\section{Subscripts}

1 One channel

$i \quad$ Inlet conditions

tot Total channels

\section{References}

1. Bejan, A. and Sciubba, E. "The optimal spacing of parallel plates cooled by forced convection", International Journal of Heat and Mass Transfer, 35, pp. 3259-3264 (1992).

2. Wei, X., and Joshi, Y. "Optimization study of stacked micro-channel heat sinks for micro-electronic cooling", IEEE Transactions Components Packaging Technologies, 26(1), pp. 156-169 (2003).

3. Bejan, A., Fowler, A.J. and Stanescu, D. "The optimal spacing between horizontal cylinders in a fixed volume cooled by natural convection", International Journal of Heat and Mass Transfer, 38, pp. 2047-2055 (1995).

4. Goshayeshi, H.R. and Ampofo, F. "Heat transfer by natural convection from a vertical and horizontal surfaces using vertical fins", Energy Power Engineering, 1(2), pp. 85-89 (2009).

5. Bar-Cohen, A. and Rohsenow, W.M. "Optimal internal structure of volumes cooled by single-phase forced and natural convection", Journal of Heat Transfer, 106, pp. 106-116 (1984).

6. Buonomo, B. and Manca, O. "Natural convection slip flow in a vertical microchannel heated at uniform heat flux", International Journal of Thermal Sciences, 49, pp. 1333-1344 (2010).

7. Safikhani, H., Abbassi, A., Khalkhali, A. and Kalteh M. "Multi-objective optimization of nanofluid flow in flat tubes using CFD, artificial neural networks and genetic algorithms", Advanced Powder Technology, 25(5), pp. 1608-1617 (2014).

8. Sadafi, M.H., Hosseini, R., Safikhani, H., Bagheri, A. and Mahmoodabadi, M.J. "Multi-objective optimization of solar thermal energy storage using hybrid of particle swarm optimization, multiple crossover and mutation operator", International Journal of Engineering Transaction B, 24, pp. 367-376 (2011).

9. Khalkhali, A., Sadafi, M., Rezapour, J. and Safikhani, H. "Pareto based multi-objective optimization of solar thermal energy storage using genetic algorithms", Transactions of the Canadian Society for Mechanical Engineering, 34(3-4), p. 463 (2010).

10. Safikhani, H. and Dolatabadi, H. "Multi-objective optimization of cooling of a stack of vertical minichannels and conventional channels subjected to natural convection", Applied Thermal Engineering, 96, pp. 144-150 (2016).

11. Safikhani, H. and Eiamsa-ard, S. "Pareto based multiobjective optimization of turbulent heat transfer flow in helically corrugated tubes", Applied Thermal Engineering, 95, pp. 275-280 (2016).

12. Safikhani, H. and Eiamsa-Ard, S. "Multi-objective optimization of turbulent tube flows over diamondshaped turbulators", Heat Transfer Engineering, 37(18), pp. 1579-1584 (2016).

13. Hao, P., Wang, B. and Li, G. "Surrogate-based optimum design for stiffened shells with adaptive sampling", AIA A Journal, 50(11), pp. 2389-2407 (2012). 
14. Hao, P., Wang, B., Tian, K. and Li, G. "Optimization of curvilinearly stiffened panels with single cutout concerning the collapse load", International Journal of Structural Stability and Dynamics, 16, pp. 155-169 (2016).

15. Wang, B., Tian, K., Hao, P., Cai, Y., Li, Y. and Sun, Y. "Hybrid analysis and optimization of hierarchical stiffened plates based on asymptotic homogenization method", Composite Structures, 132, pp. 136-147 (2015).

16. Deb, K., Agrawal, S., Pratap, A. and Meyarivan, T. "A fast and elitist multi-objective genetic algorithm: NSGA-II", IEEE Trans Evolutionary Computation, 6, pp. 182-197 (2002).

17. Safikhani, H., Akhavan-Behabadi, M.A., NarimanZadeh, N. and Mahmoodabadi, M.J. "Modeling and multi-objective optimization of square cyclones using CFD and neural networks", Chemical Engineering Research and Design, 89, pp. 301-309 (2011).

18. Safikhani, H., Hajiloo, A. and Ranjbar, M.A. "Modeling and multi-objective optimization of cyclone separators using CFD and genetic algorithms", Computers and Chemical Engineering, 35, pp. 1064-1071 (2011).

19. Amanifard, N., Nariman-Zadeh, N., Borji, M., Khalkhali, A. and Habibdoust, A. "Modeling and Pareto optimization of heat transfer and flow coefficients in micro channels using GMDH type neural networks and genetic algorithms", Energy Conversion and Management, 49, pp. 311-325 (2008).

20. Bejan, A., Convection Heat Transfer, Wiley (2004).

\section{Biographies}

Hamed Safikhani is Assistant Professor of Mechanical Engineering at Arak University, Iran. He received his $\mathrm{PhD}$ degree from Amirkabir University of Technology in 2014. He is one of the members of "The Promised SORAYYA Technologist" sciencebased industries in Iran. He has co-authored more than 40 journal and conference publications. His research interests include two-phase and single-phase convective heat transfer in macro-micro and nanoscales. He is currently working on augmentation of heat transfer by different passive techniques in two-phase flow and nanofluid single-phase flow.

Zahra Shirazi was born in 1992 in Isfahan, Iran. She received the BSc degree in Mechanical Engineering from Arak University in 2015. The title of her BSc dissertation was "Numerical Study of Nanofluid Flow in Pipes" under the supervision of Dr. Hamed Safikhani. She is currently working on augmentation of heat transfer by different passive techniques in singlephase flow systems. 